\title{
let7f-5p attenuates inflammatory injury in in vitro pneumonia models by targeting MAPK6
}

\author{
LIN XU ${ }^{1,2}$, QINGYING SONG ${ }^{3}$, ZHANGHONG OUYANG $^{4}$, XIANGYAN ZHANG $^{1,2}$ and CHENG ZHANG ${ }^{1,2}$ \\ ${ }^{1}$ Department of Biomedical Science, Guizhou University Medical College, Guiyang, Guizhou 550025; \\ ${ }^{2}$ Department of Respiratory and Critical Medicine, Guizhou Provincial People's Hospital, Guiyang, Guizhou 550002; \\ ${ }^{3}$ Department of Anesthesiology, The Second Affiliated Hospital of Guizhou College of Traditional Chinese Medicine, \\ Guiyang, Guizhou 550003; ${ }^{4}$ Development Planning Division, Guizhou Provincial People's Hospital, \\ Guiyang, Guizhou 550002, P.R. China
}

Received June 29, 2020; Accepted October 19, 2020

DOI: $10.3892 / \mathrm{mmr} .2020 .11734$

\begin{abstract}
Pneumonia accounts for $\sim 1.3$ million mortalities in children per year worldwide. MicroRNAs are implicated in several diseases, including cancer and pneumonia; however, the role of let $7 \mathrm{f}-5 \mathrm{p}$ in pneumonia is not completely understood. In the present study, lipopolysaccharide (LPS) was used to establish an in vitro pneumonia model in A549 and WI-38 cells. The reverse transcription-quantitative PCR (RT-qPCR) and western blotting results demonstrated that let7f-5p expression levels were significantly decreased, whereas MAPK6 expression levels were significantly increased in the peripheral venous blood of patients with pneumonia and in LPS-induced A549 and WI-38 cells compared with healthy volunteers and control cells, respectively. Furthermore, the dual-luciferase reporter assay demonstrated that let $7 \mathrm{f}-5 \mathrm{p}$ targeted the 3'-untranslated region of MAPK6. The ELISA and RT-qPCR results demonstrated that let $7 \mathrm{f}-5 \mathrm{p}$ mimic ameliorated LPS-induced inflammatory injury in A549 and WI-38 cells, as demonstrated by decreased expression levels of proinflammatory cytokines, including TNF- $\alpha$ and IL-6. In addition, the Cell Counting Kit- 8 assay results indicated that let7f-5p mimic ameliorated LPS-induced reductions in cell viability, and the western blotting results demonstrated that let $7 \mathrm{f}-5 \mathrm{p}$ mimic reversed LPS-induced activation of the STAT3 signaling pathway. Notably, the aforementioned let $7 \mathrm{f}-5 \mathrm{p}$-mediated effects were reversed by MAPK6 overexpression. Collectively, the results of the present study suggested that let7f-5p inhibited inflammation by targeting MAPK6 in the in vitro pneumonia
\end{abstract}

Correspondence to: Dr Xiangyan Zhang or Dr Cheng Zhang, Department of Respiratory and Critical Medicine, Guizhou Provincial People's Hospital, 52 Zhongshan East Road, Guiyang, Guizhou 550002, P.R. China

E-mail: zxy35762@126.com

E-mail: zhangchenggzpph@126.com

Key words: pneumonia, let7f-5p, MAPK6, STAT3 signaling pathway model, thus let $7 \mathrm{f}-5 \mathrm{p}$ may serve as a potential novel therapeutic target for pneumonia.

\section{Introduction}

Pneumonia is a lower respiratory illness, which is characterized by the following symptoms: Cough, fever, chest pain and in severe cases, heart failure (1). Pneumonia is a common infectious disease, with high morbidity rates $(\sim 0.02 \%)$ worldwide, particularly in children $(2,3)$. Inflammation is a key defense mechanism to injury that prevents the entry of hazardous substances into the body; however, inflammation also displays the potential to cause injury (4). The respiratory tract is easily infected on account of poor immune function in pediatric cases (5). Inflammation from endotoxins is a leading cause of pneumonia (6). As a potent endotoxin for inflammation, lipopolysaccharide (LPS) is the primary bioactive component of the cell wall of gram-negative bacterium (7). Therefore, developing novel therapeutic strategies to inhibit the progression of pneumonia is important.

MicroRNAs (miRNAs/miRs) are a subgroup of non-coding RNAs $\leq 200$ nucleotides in length, which control gene expression at the transcriptional and post-transcriptional levels, thus affecting cellular processes (8). miRNAs, such as miR-3941, have been reported to regulate inflammatory responses and diseases (9). In addition, certain miRNAs have been identified in the pathogenesis of pneumonia. For example, Abd-El-Fattah et al (10) demonstrated that miR-155, miR-21 and miR-197 are upregulated in patients with pneumonia, but their targets and respective functions have not been identified. In 2017, Huang et al (11) identified miRNA biomarkers for pneumonia via RNA-sequencing and bioinformatics analysis, which demonstrated that let7f is downregulated in the peripheral blood of patients with severe pneumonia. Therefore, the present study aimed to investigate the role of let $7 \mathrm{f}$ in pneumonia.

\section{Materials and methods}

Serum samples. As pneumonia is also frequent in children (2), the present study aimed to identify a potential therapeutic target for pneumonia in children. Peripheral venous blood $(3 \mathrm{ml})$ 
was collected from 29 healthy children (22 male patients and 7 female patients; age range, 1-8 years; mean age, 4.7 years) and 29 patients with pneumonia (22 male patients and 7 female patients; age range, 1-7 years; mean age, 3.8 years) at Guizhou Provincial People's Hospital (Guiyang, China) between March 2016 and February 2018. The exclusion criteria were as follows: i) Patients with immunodeficiency, tuberculosis infection or asthma; and ii) patients with respiratory tract infection or inflammatory disease. Patients aged 1-8 years who were diagnosed with pneumonia were included in the present study. Blood samples were centrifuged at $1,000 \mathrm{x}$ g for $10 \mathrm{~min}$ at $4^{\circ} \mathrm{C}$ and the supernatant was collected for subsequent experimentation. The present study was approved by the Ethical Committee of Guizhou Provincial People's Hospital (approval no. 2016011605). The parents or legal guardians of all participants provided written informed consent.

Cell culture. The human lung adenocarcinoma A549 cell line and normal human fibroblast WI-38 cell line were purchased from American Type Culture Collection. Cells were maintained in DMEM supplemented with $10 \%$ FBS and $1 \%$ penicillin/streptomycin (all purchased from Invitrogen; Thermo Fisher Scientific, Inc.) at $37^{\circ} \mathrm{C}$ with $5 \% \mathrm{CO}_{2}$.

Cell transfection. The full length of MAPK6 was reconstructed into a pcDNA3.1 empty vector (Invitrogen; Thermo Fisher Scientific, Inc.). let7f-5p mimic (5'-UUGAUAUGUUAGAUG AUGGAGU-3') and negative control (NC) mimic (5'-UCA CAACCUCCUAGAAAGAGUAGA-3') were synthesized by Shanghai GenePharma Co., Ltd. A549 and WI-38 cells (1x10 4 cells/well) were transfected with $2 \mu \mathrm{g}$ pcDNA3.1, $2 \mu \mathrm{g}$ pcDNA3.1-MAPK6, $100 \mathrm{nM}$ NC mimic or $100 \mathrm{nM}$ let7f-5p mimic using Lipofectamine ${ }^{\circledR} 2000$ reagent (Invitrogen; Thermo Fisher Scientific, Inc.) according to the manufacturer's protocol. After transfection for $48 \mathrm{~h}$ at $37^{\circ} \mathrm{C}$, cells were used for subsequent experiments.

LPS treatment. Briefly, cells were seeded ( $2 \times 10^{5}$ cells/well) into 6-well plates and cultured at $37^{\circ} \mathrm{C}$ for $24 \mathrm{~h}$. To establish the in vitro pneumonia model, WI-38 cells were incubated with $10 \mu \mathrm{g} / \mathrm{ml}$ LPS (Beijing Solarbio Science \& Technology Co., Ltd.) for $12 \mathrm{~h}$ at $37^{\circ} \mathrm{C}$ as previously described $(12,13)$, whereas A549 cells were incubated with $10 \mu \mathrm{g} / \mathrm{ml} \mathrm{LPS} \mathrm{for} 12 \mathrm{~h}$ at $37^{\circ} \mathrm{C}$ as previously described (14).

Reverse transcription-quantitative PCR (RT-qPCR). Total RNA was extracted from peripheral venous blood, and A549 and WI-38 cells using TRIzol ${ }^{\circledR}$ reagent (Invitrogen; Thermo Fisher Scientific, Inc.). Total RNA was reverse transcribed into cDNA using the PrimeScript ${ }^{\mathrm{TM}}$ RT reagent kit (cat. no. RR047A; Takara Biotechnology Co., Ltd.), according to the manufacturer's protocol. Subsequently, qPCR was performed using the SYBR Premix Ex Taq (cat. no. RR003A; Takara Biotechnology Co.,Ltd.). The following thermocycling conditions were used for qPCR: Initial denaturation at $94^{\circ} \mathrm{C}$ for $5 \mathrm{~min}$; followed by 40 cycles of degeneration at $94^{\circ} \mathrm{C}$ for $30 \mathrm{sec}$, annealing at $60^{\circ} \mathrm{C}$ for $30 \mathrm{sec}$ and extension at $72^{\circ} \mathrm{C}$ for $1 \mathrm{~min}$. The following primers were used for qPCR: let7f-5p forward, 5'-TTGATATGTTAGATGATG GAGT-3' and reverse, 5'-ACTCCATCATCTAACATATCAA-3'; IL-6 forward, 5'-AGCCACTCACCTCTTCAGAACGAA-3' and reverse, 5'-TACTCATCTGCACAGCTCTGGCTT-3'; TNF- $\alpha$ forward, 5'-GCCAATGGCATGGATCTCAAAG-3' and reverse, 5'-CAGAGCAATGACTCCAAAGT-3'; U6 forward, 5'-GTG CTCGCTTCGGCAGCACAT-3' and reverse, 5'-AATATGGAA CGCTTCACGAAT-3'; MAPK6 forward, 5'-GTACACATGTGT TATCTACCTCA-3 and reverse, 5'-TACAATAAACGCTGG CTAA-3'; and GAPDH forward, 5'-GCACCGTCAAGGCTG AGAAC-3' and reverse, 5'-TGGTGAAGACGCCAGTGGA-3'. let7f-5p and IL-6/TNF- $\alpha$ /MAPK6 mRNA expression levels were quantified using the $2^{-\Delta \Delta \mathrm{Cq}}$ method (15) and normalized to the internal reference genes U6 and GAPDH, respectively.

Western blotting. Total protein was extracted from peripheral venous blood, and A549 and WI-38 cells using RIPA lysis buffer (Sigma-Aldrich; Merck KGaA) supplemented with protease inhibitors. Total protein was quantified using the BCA method (Beyotime Institute of Biotechnology). Equal amounts of protein (15 $\mu \mathrm{g} /$ lane) were separated via $8 \%$ SDS-PAGE and transferred onto PVDF membranes. Following blocking with $5 \%$ non-fat milk at room temperature for $2 \mathrm{~h}$, the membranes were incubated overnight at $4^{\circ} \mathrm{C}$ with primary antibodies (all purchased from Cell Signaling Technology, Inc.) targeted against: MAPK6 (cat. no. 4067; 1:1,000), STAT3 (cat. no. 12640; 1:1,000), phosphorylated-STAT3 (cat. no. 9145; 1:1,000) and GAPDH (cat. no. 5174; 1:1,000). Following washing three times with TBST, the membranes were incubated with HRP-conjugated goat anti-rabbit secondary antibodies (cat. no. 7074; 1:3,000; Cell Signaling Technology, Inc.) at room temperature for $1 \mathrm{~h}$. Protein bands were visualized using an ECL system (Beyotime Institute of Biotechnology). Protein expression was semi-quantified using ImageJ software (version 1.50; National Institutes of Health).

Cell viability assay. A549 and WI-38 cell viability were assessed using the Cell Counting Kit-8 (CCK-8) detection kit (cat. no. CSP04; Dojindo Molecular Technologies, Inc.) according to the manufacturer's protocol. Briefly, cells were seeded $\left(5 \times 10^{3}\right.$ cells/well) into 96 -well plates and cultured for $24 \mathrm{~h}$ at $37^{\circ} \mathrm{C}$. Subsequently, $20 \mu \mathrm{lCCK}-8$ solution was added to each well for $1 \mathrm{~h}$ at $37^{\circ} \mathrm{C}$. The absorbance was measured at a wavelength of $450 \mathrm{~nm}$ using a microplate reader.

ELISA. A549 and WI-39 cell culture media were collected. The levels of inflammatory cytokines, including IL-6 (cat. no. D6050) and TNF- $\alpha$ (cat. no. MTA00B), were detected using ELISA kits (R\&D Systems, Inc.) according to the manufacturer's protocol.

Dual-luciferase reporter assay. TargetScan software (version 7.1; www.targetscan.org/vert_71) was used to predict the complementary binding sites between let $7 \mathrm{f}-5 \mathrm{p}$ and the 3'-untranslated region (UTR) of MAPK6. To validate the interaction between let7f-5p and MAPK6, A549 and WI-38 cells were seeded $\left(1 \times 10^{4}\right.$ cells/well) into 24 -well plates and co-transfected with wild-type (WT) MAPK6 (0.2 mg) or mutant MAPK6 (0.2 mg) pmiRGLO dual-luciferase vectors (Promega Corporation) and let7f-5p mimic (20 nM) or NC mimic (20 nM) using Lipofectamine 2000. Following incubation for $24 \mathrm{~h}$ at $37^{\circ} \mathrm{C}$, luciferase activities were detected using a Dual-Luciferase Reporter assay system (Promega 

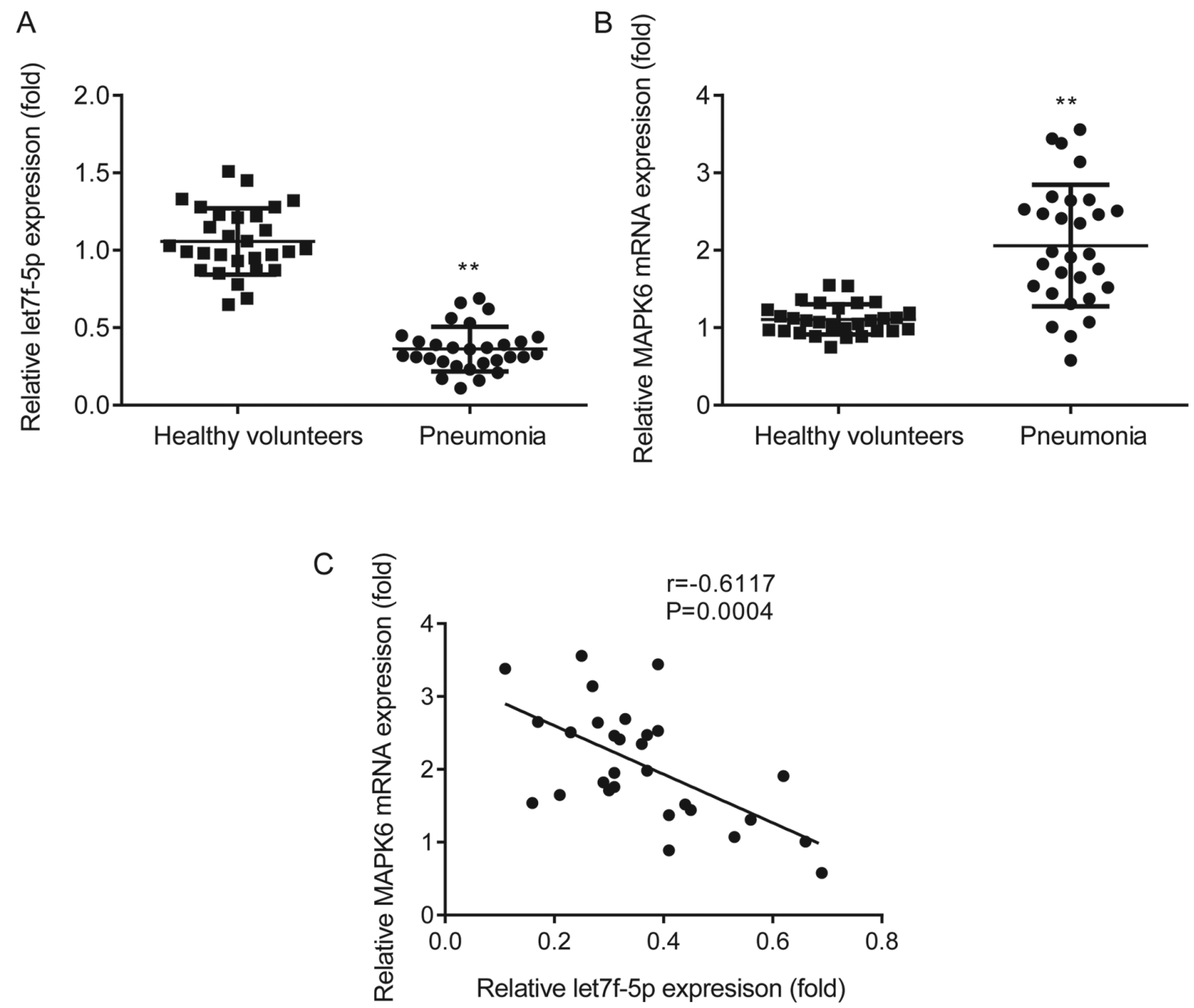

Figure 1. let7f-5p expression is negatively correlated with MAPK6 expression in patients with pneumonia. Reverse transcription-quantitative PCR analysis was performed to detect the expression levels of (A) let7f-5p and (B) MAPK6 in the peripheral venous blood of patients with pneumonia and healthy volunteers. (C) Pearson's correlation analysis was performed to determine the correlation between let7f-5p and MAPK6. ${ }^{* *} \mathrm{P}<0.01$ vs. healthy volunteers.

Corporation) according to the manufacturer's protocol. Firefly luciferase activity was normalized to Renilla luciferase activity.

Statistical analysis. Statistical analyses were performed using GraphPad software (version 6.0; GraphPad Software, Inc.). All experiments were performed in triplicate. Data are presented as the mean \pm SD. The unpaired Student's t-test was performed to compare the difference of let7f-5p and MAPK6 between healthy volunteers and patients with pneumonia. The unpaired Student's t-test was performed to compare differences between two groups for in vitro experiments. One-way ANOVA followed by Tukey's post hoc test was used to compare differences among multiple groups. Pearson's correlation analysis was performed to determine the correlation between let7f-5p and MAPK6 in the peripheral venous blood of patients with pneumonia. $\mathrm{P}<0.05$ was considered to indicate a statically significant difference.

\section{Results}

let7f-5p expression is negatively correlated with MAPK6 expression in patients with pneumonia. The expression profiles of let7f-5p and MAPK6 in patients with pneumonia and healthy volunteers were assessed via RT-qPCR. let7f-5p expression was significantly decreased (Fig. 1A) and MAPK6 expression was significantly increased (Fig. 1B) in the peripheral venous blood of patients with pneumonia compared with healthy volunteers. Pearson's correlation analysis demonstrated that let7f-5p expression was negatively correlated with MAPK6 expression in patients with pneumonia (Fig. 1C).

LPS-induced inflammatory injury in A549 and WI-38 cells. The effects of LPS on A549 and WI-38 cells were assessed by performing CCK- 8 and ELISA assays. The CCK- 8 assay results indicated that LPS treatment significantly decreased cell viability compared with the control group (Fig. 2A). In addition, the RT-qPCR and ELISA results indicated that LPS treatment significantly increased the expression and release of proinflammatory cytokines TNF- $\alpha$ and IL- 6 in A549 and WI-38 cells compared with the control group (Fig. 2B-E). Collectively, the results suggested that LPS induced inflammatory injury in A549 and WI-38 cells.

let7f-5p and MAPK6 expression in LPS-induced inflammatory injury. The expression profiles of let7f-5p and MAPK6 in the 

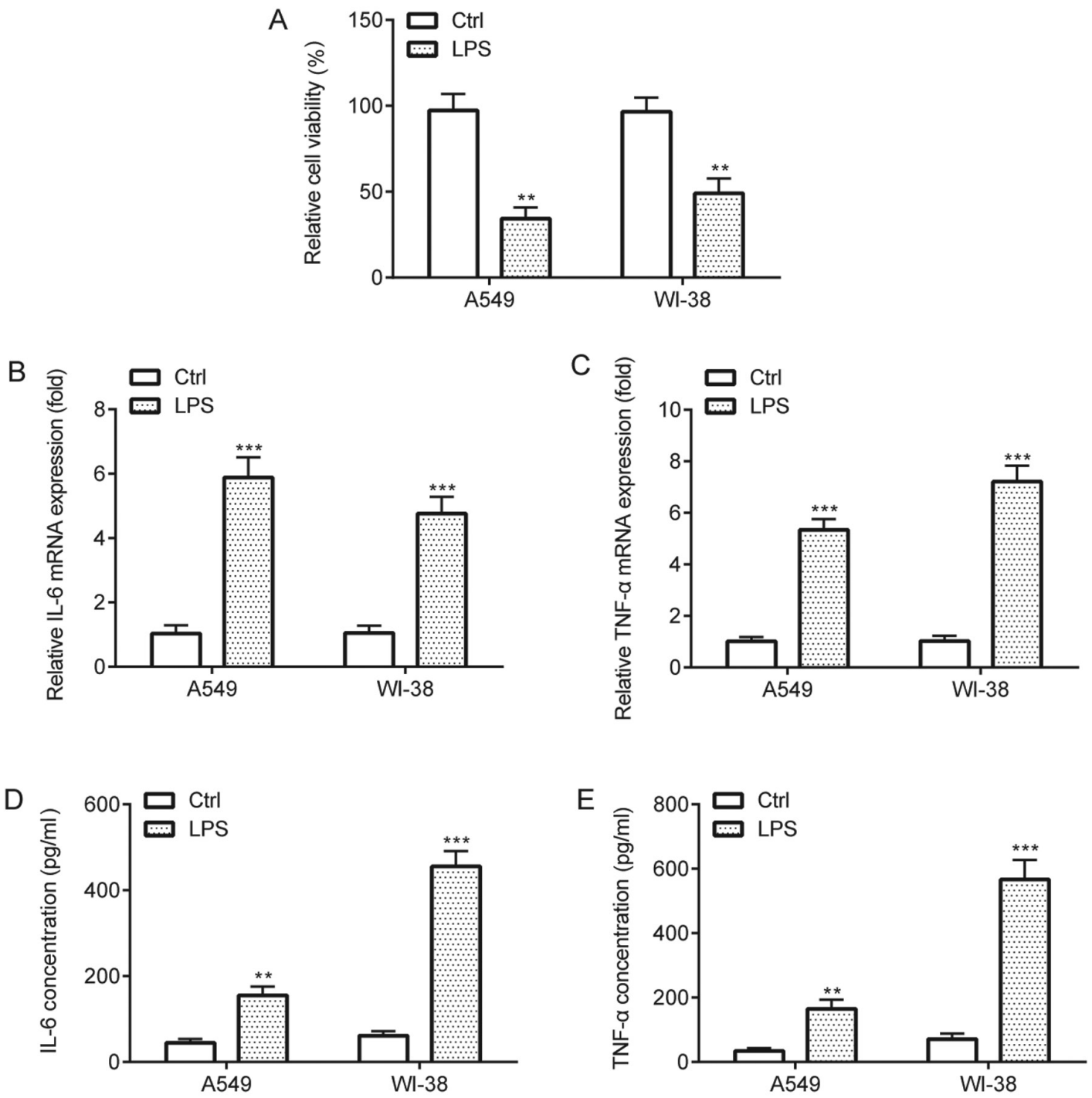

Figure 2. LPS-induced inflammatory injury in A549 and WI-38 cells. (A) Cell Counting Kit-8 assay was performed to assess cell viability in A549 and WI-38 cells. Reverse transcription-quantitative PCR was performed to measure (B) IL-6 and (C) TNF- $\alpha$ mRNA expression levels in A549 and WI-38 cells. ELISAs were performed to assess the concentrations of (D) IL-6 and (E) TNF- $\alpha$ in the cell culture medium of A549 and WI-38 cells. ${ }^{* *} \mathrm{P}<0.01$ and ${ }^{* * * *} \mathrm{P}<0.001$ vs. Ctrl. LPS, lipopolysaccharide; Ctrl, control.

in vitro pneumonia model in A549 and WI-38 cells were assessed via RT-qPCR and western blotting. The results demonstrated that let7f-5p expression levels were significantly lower (Fig. 3A), whereas MAPK6 protein expression levels were significantly higher (Fig. 3B and C) in LPS-treated A549 and WI-38 cells compared with the control group.

let $7 f-5 p$ targets MAPK6. The interaction between let7f-5p and MAPK6 was verified by performing the dual-luciferase reporter assay. TargetScan software identified complementary binding sites between let7f-5p and the 3'UTR of MAPK6 (Fig. 4A). The results demonstrated that let7f-5p mimic significantly increased let7f-5p expression in A549 and WI-38 cells compared with NC mimic (Fig. 4B). The dual-luciferase reporter assay results indicated that compared with NC mimic, let7f-5p mimic significantly decreased the luciferase activity of WT MAPK6 in A549 and WI-38 cells, but did not significantly alter the luciferase activity of MUT MAPK6 in A549 or WI-38 cells (Fig. 4C and D). let7f-5p attenuates LPS-induced inflammatory injury by targeting MAPK6. The effects of let7f-5p and MAPK6 in the LPS-induced in vitro pneumonia model were investigated in A549 and WI-38 cells. The RT-qPCR and western blotting results demonstrated that pcDNA3.1-MAPK6 significantly increased MAPK6 mRNA and protein expression levels (Fig. 5A-C) compared with pcDNA3.1 in A549 and WI-38 cells. The CCK-8 assay results suggested that let7f-5p mimic significantly ameliorated LPS-induced reductions in cell viability in A549 and WI-38 cells, but let7f-5p mimic-mediated effects were significantly reversed by MAPK6 overexpression (Fig. 6A). The RT-qPCR and ELISA results demonstrated that let7f-5p mimic significantly ameliorated LPS-induced TNF- $\alpha$ and IL-6 expression and release in A549 and WI-38 cells, which was also significantly reversed by transfection with pcDNA3.1-MAPK6 (Fig. 6B-E).

STAT3 is involved in let7f-5p/MAPK6-mediated regulation of LPS-induced inflammatory injury. Compared with the control group, LPS significantly increased STAT3 activation 

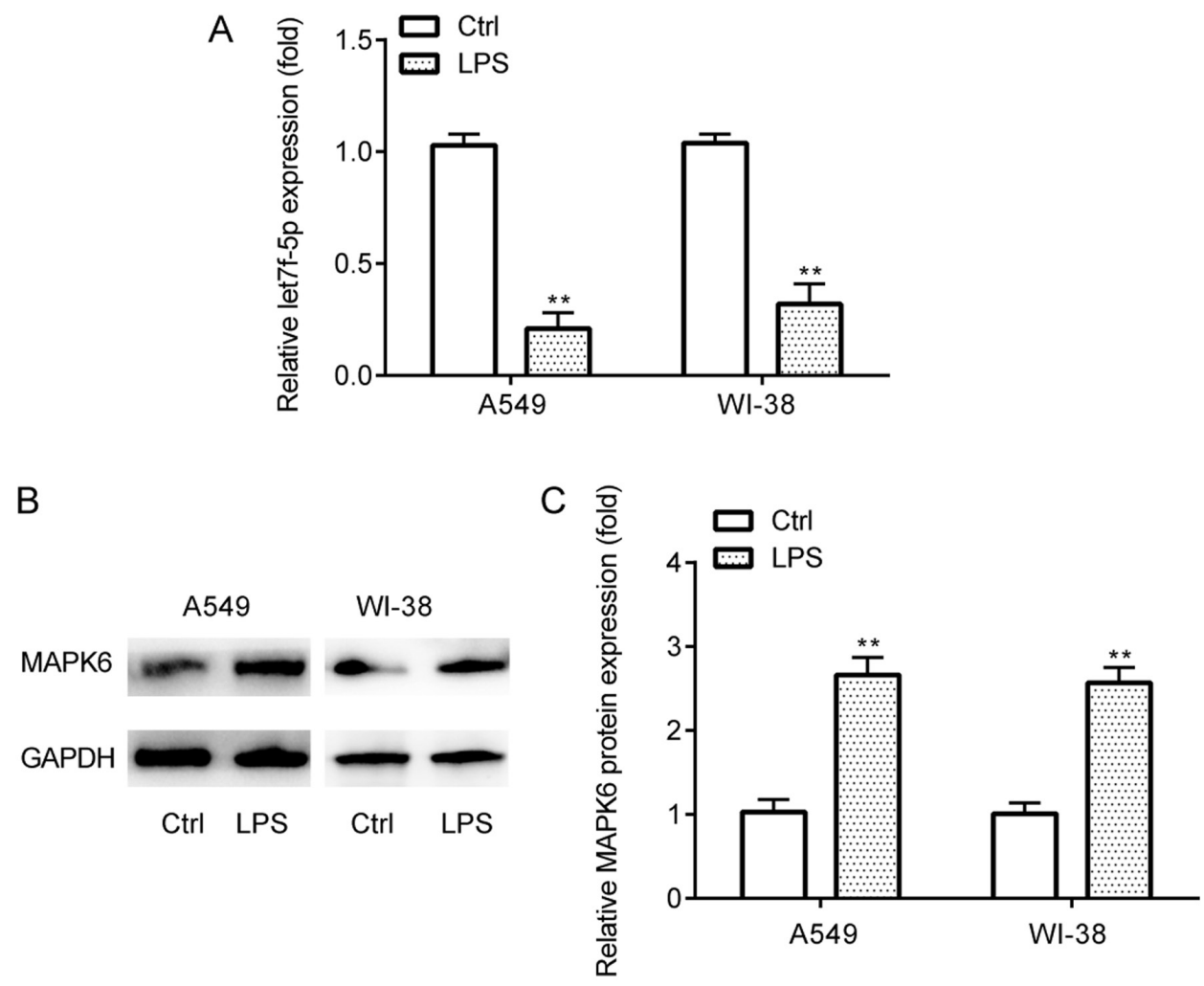

Figure 3. let7f-5p and MAPK6 expression in LPS-induced inflammatory injury. (A) Reverse transcription-quantitative PCR was performed to detect let7f-5p expression levels in A549 and WI-38 cells. MAPK6 protein expression levels were (B) determined via western blotting and (C) semi-quantified in A549 and WI-38 cells. ${ }^{* *} \mathrm{P}<0.01$ vs. Ctrl. LPS, lipopolysaccharide; Ctrl, control.

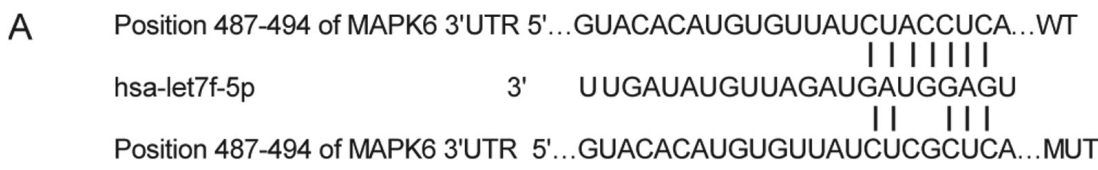
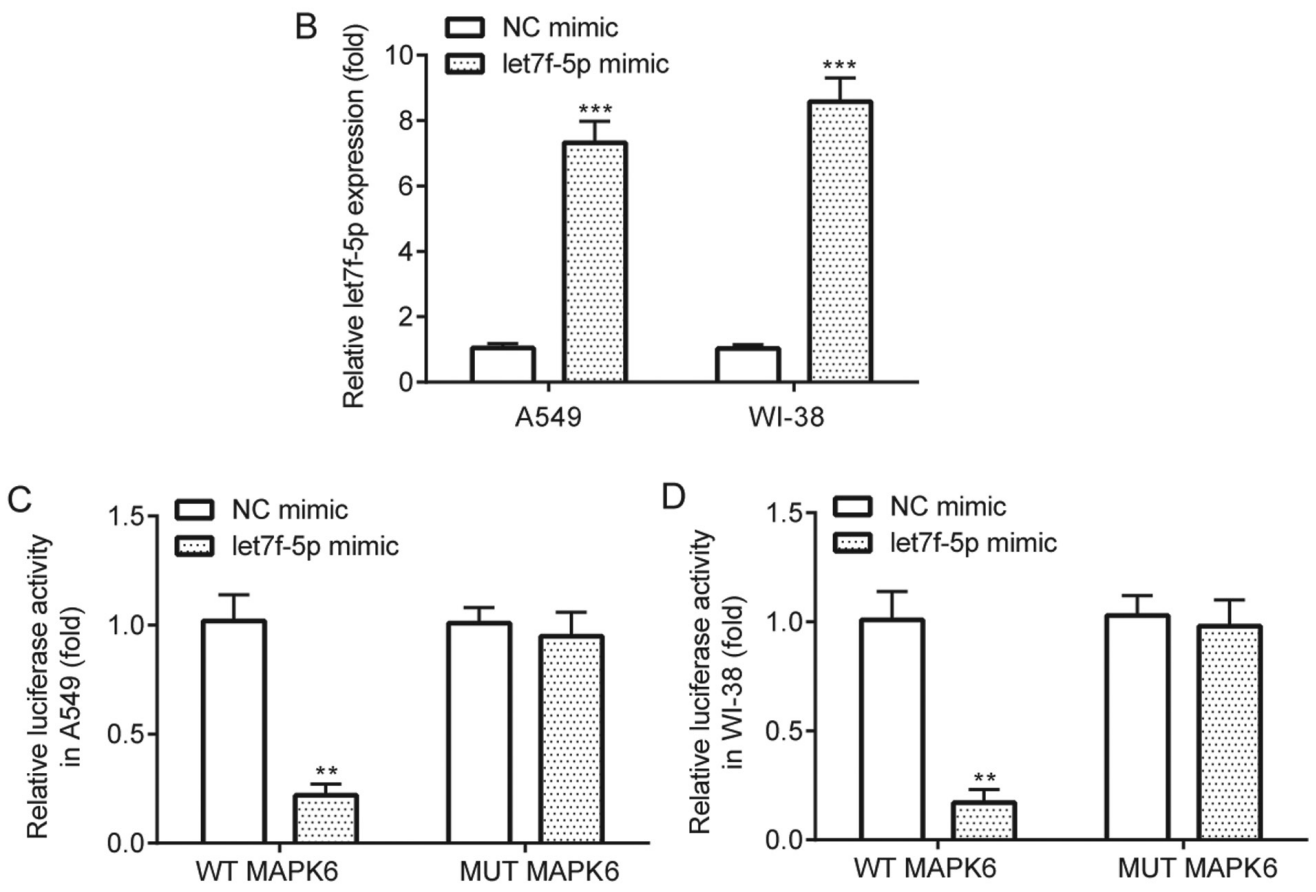

Figure 4. let7f-5p targets MAPK6. (A) TargetScan software was used to predict the complementary binding sites between let7f-5p and MAPK6. (B) Reverse transcription-quantitative PCR was performed to assess the transfection efficiency of let7f-5p mimic in A549 and WI-38 cells. Dual-luciferase reporter assays were performed to validate the interaction between let7f-5p and MAPK6 in (C) A549 and (D) WI-38 cells. ${ }^{* *} \mathrm{P}<0.01$ and $^{* * * *} \mathrm{P}<0.001$ vs. NC mimic. NC, negative control; UTR, untranslated region; WT, wild-type; MUT, mutant. 

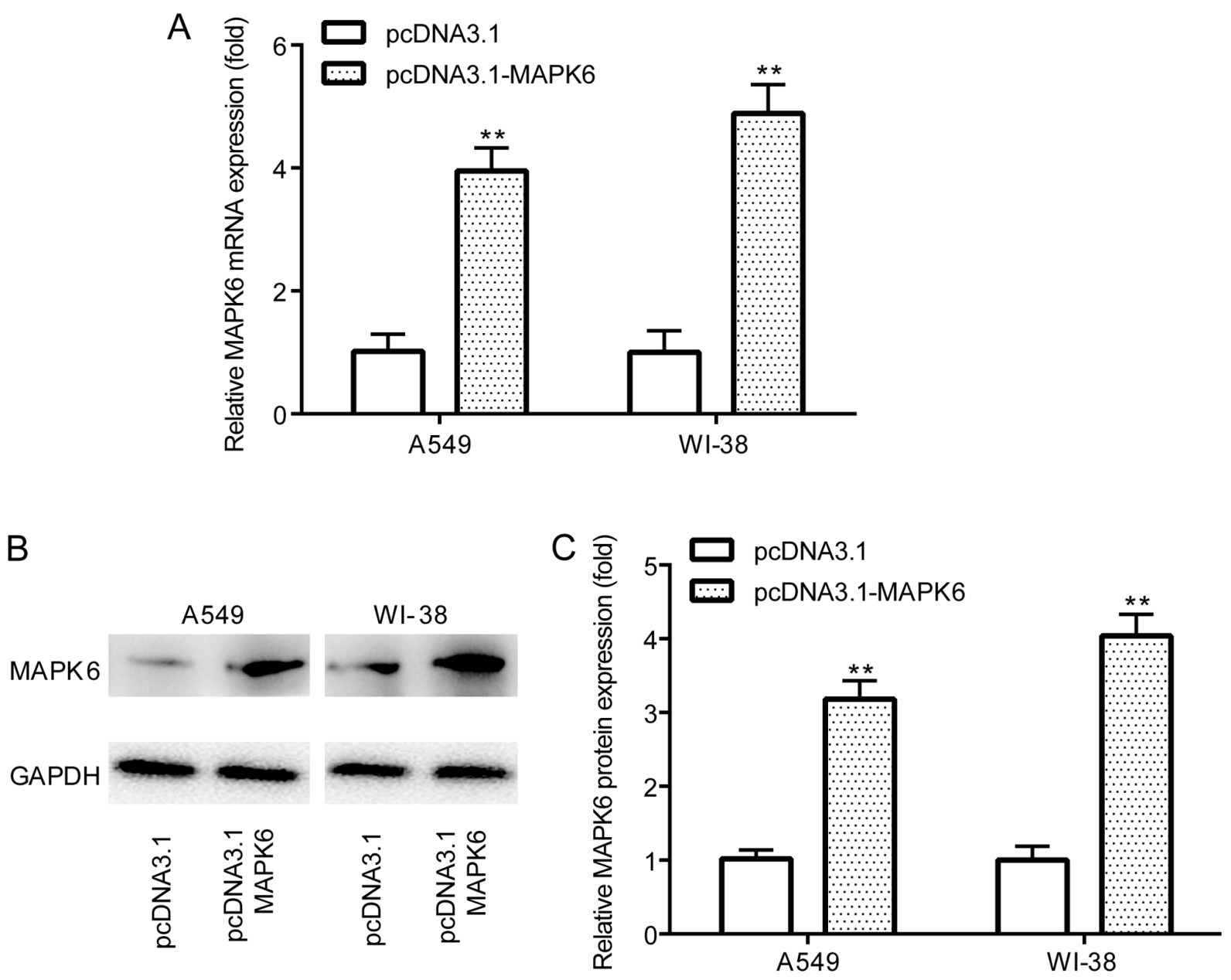

Figure 5. pcDNA3.1-MAPK6 increases MAPK6 expression. (A) Reverse transcription-quantitative PCR was performed to assess the transfection efficiency of pcDNA3.1-MAPK6 in A549 and WI-38 cells. pcDNA3.1-MAPK6 transfection efficiency in A549 and WI-38 cells was also (B) determined via western blotting and $(\mathrm{C})$ semi-quantified. ${ }^{* *} \mathrm{P}<0.01$ vs. pcDNA3.1.

in A549 and WI-38 cells, which was reversed by transfection with let7f-5p mimic (Fig. 7A and B). However, let7f-5p mimic-mediated effects on STAT3 activation were significantly reversed by transfection with pcDNA3.1-MAPK6.

\section{Discussion}

Pneumonia is a common infectious disease, with high mortality and morbidity rates worldwide $(2,3)$; therefore, developing novel therapeutic strategies for the management of pneumonia is important. Targeted therapy is extensively applied in the treatment of several diseases (16). For example, the let 7 family participates in multiple carcinogenic signaling pathways (17). let-7 serves as a tumor suppressor, whereby let-7 downregulation promotes lung cancer cell proliferation (18), whereas let-7b and let-7c are crucial for lung restoration in influenza pneumonia model mice (18). let7f inhibition facilitates neuroprotection in ischemic stroke (19), and let7f expression is elevated following ischemia-reperfusion (20), but decreased in patients with papillary thyroid cancer (21). By performing RNA-sequencing and bioinformatics analysis, a previous study demonstrated that let7f expression is decreased in the peripheral blood of patients with severe pneumonia compared with healthy volunteers (11). More recently, it has been reported that let7f expression is decreased in the blood of extracellular vesicles of alcohol-drinkers without liver injury compared with non-drinkers (22). To the best of our knowledge, the present study was the first to investigate the role of let7f-5p in pneumonia.

MAPK6 serves as a promoter of several diseases: Small nucleolar RNA host gene 6 facilitates breast cancer cell proliferation and metastasis by regulating the miR-26a-5p/MAPK6 axis (23); miR-144-3p inhibits the progression of cervical cancer by targeting MAPK6 (24); nuclear paraspeckle assembly transcript 1 enhances myocardial ischemia-reperfusion injury via the miR-495-3p/MAPK6 axis (25); and miR-26a-5p negatively regulates neuropathic pain by targeting MAPK6 (26). As for the function of MAPK6 in inflammation, hierarchical clustering in tongue tissue with hyperplasia suggests that cytokine-mediated inflammation may be associated with MAPK6 (27). However, the exact role of MAPK6 in pneumonia is not completely understood.

The present study aimed to investigate the role of let7f-5p and MAPK6 by recruiting healthy volunteers and patients with pneumonia, and using in vitro pneumonia model. The results demonstrated that let7f-5p expression was significantly decreased, whereas MAPK6 expression was significantly increased in the peripheral venous blood of patients with 

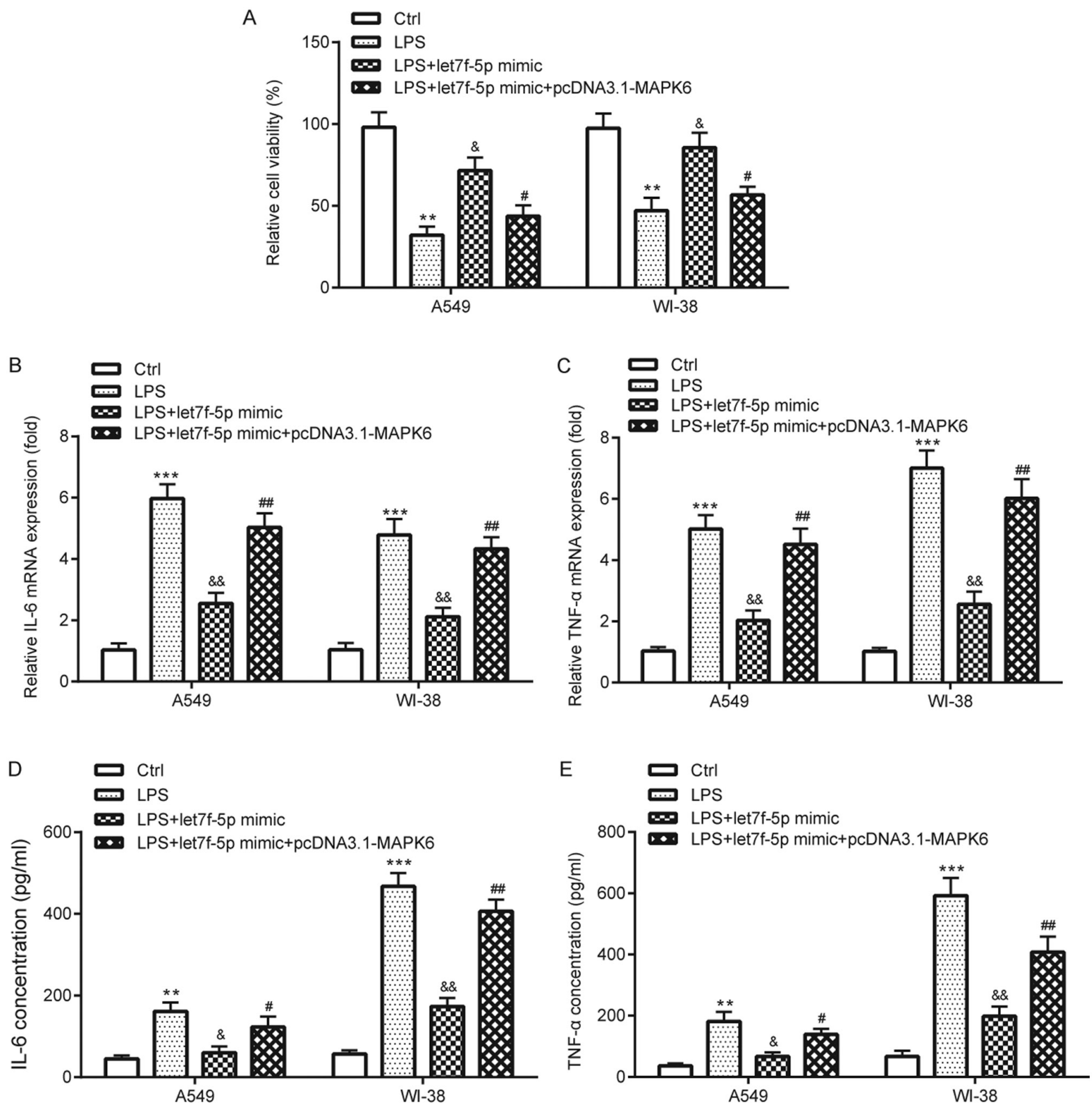

Figure 6. let7f-5p attenuates LPS-induced inflammatory injury by targeting MAPK6. (A) Cell Counting Kit-8 assay was performed to assess cell viability in A549 and WI-38 cells. Reverse transcription-quantitative PCR was performed to determine (B) IL-6 and (C) TNF- $\alpha$ mRNA expression levels in A549 and WI-38 cells. ELISAs were performed to assess the concentrations of (D) IL-6 and (E) TNF- $\alpha$ in the cell culture medium of A549 and WI-38 cells. ${ }^{* *} \mathrm{P}<0.01$ and ${ }^{* * * *} \mathrm{P}<0.001$ vs. Ctrl; ${ }^{\&} \mathrm{P}<0.05$ and ${ }^{\& \&} \mathrm{P}<0.01$ vs. LPS; ${ }^{*} \mathrm{P}<0.05$ and ${ }^{\# *} \mathrm{P}<0.01$ vs. LPS + let7f-5p mimic. LPS, lipopolysaccharide; Ctrl, control.

A

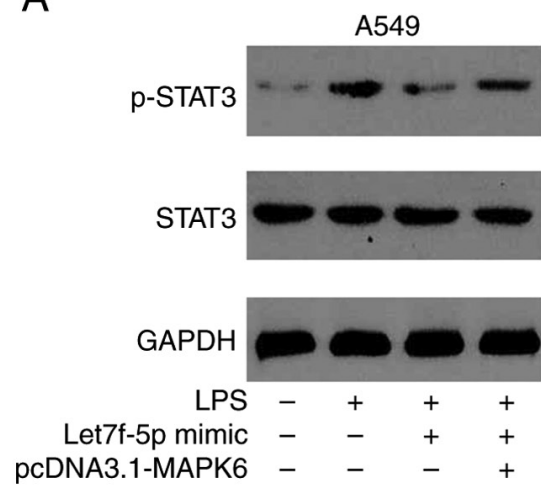

WI-38

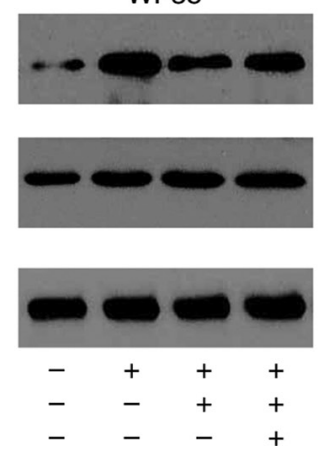

B 口 Ctrl

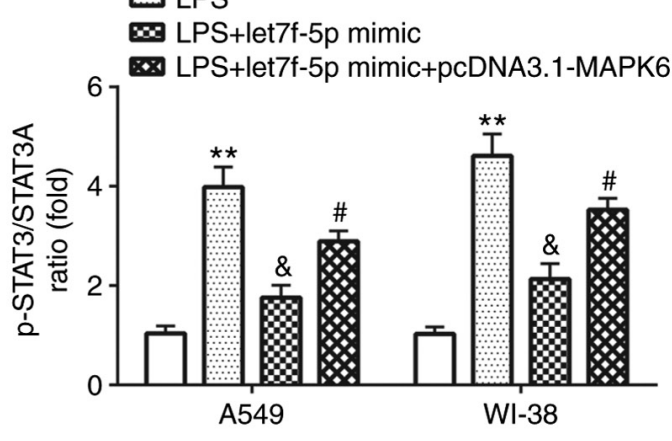

Figure 7. STAT3 is involved in let7f-5p/MAPK6-mediated regulation of LPS-induced inflammatory injury. STAT3 protein expression levels were (A) determined by western blotting and (B) semi-quantified in A549 and WI-38 cells. ${ }^{* * *} \mathrm{P}<0.01$ vs. Ctrl; ${ }^{\&} \mathrm{P}<0.05$ vs. LPS; ${ }^{*} \mathrm{P}<0.05$ vs. LPS + let7f-5p mimic. LPS, lipopolysaccharide; Ctrl, control; p, phosphorylated. 
pneumonia and in LPS-induced WI-38 and A549 cells compared with healthy volunteers and control cells, respectively. The results also demonstrated that let7f-5p targeted MAPK6 in WI-38 and A549 cells, and let7f-5p expression was negatively correlated with MAPK6 expression in the peripheral venous blood of patients with pneumonia.

Pneumonia is associated with inflammation (6). TNF- $\alpha$, a predominant cytokine that is produced by monocytes and macrophages, is an important inflammatory mediator $(28,29)$. TNF- $\alpha$ initiates the inflammatory response by inducing local infiltration, neutrophil chemotaxis, phagocytosis and killing of pathogens (30). IL-6, an important cytokine that is produced by monocytes, macrophages and lymphocytes, is a pivotal mediator during the acute phase of inflammatory response $(28,30)$. The results of the present study demonstrated that, compared with the control group, LPS treatment significantly increased the expression levels of TNF- $\alpha$ and IL-6 in A549 and WI-38 cells, which were reversed by transfection with let7f-5p mimic. However, let7f-5p mimic-mediated effects were reversed by MAPK6 overexpression.

The STAT3 signaling pathway is pivotal for the inflammatory response (31). During acute inflammatory injury, the STAT3 signaling pathway is implicated in lung injury (32) and LPS-induced inflammatory injury, as well as in the circular RNA (circ)_0038467/miR-338-3p axis (33). The results of the present study demonstrated that LPS significantly increased STAT3 activation compared with the control group, which was reversed by transfection with let7f-5p mimic. Moreover, let7f-5p mimic-mediated effects on STAT3 activation were reversed by MAPK6 overexpression.

As for the association between the let7f-5p/MAPK6/STAT3 axis and inflammation, several reports in other inflammation-related diseases have been published. In 2019, Tan et al (34) reported that let7f-5p attenuated inflammation in systemic lupus erythematosus by targeting NLR family pyrin domain containing 3. In 2020, Yao et al (35) demonstrated that MAPK6 was involved in circ_0000285-induced inflammation in diabetic nephropathy. The STAT3 signaling pathway is pivotal for the inflammatory response (31). In 2019, Li et al (36) reported that let7f-5p reduced Th17 differentiation in multiple sclerosis by targeting STAT3. In 2018, Kim et al (37) demonstrated that orientin repressed breast cancer cell invasion via the MAPK6/STAT3 signaling pathway (37). However, the relationship between let7f-5p and MAPK6, as well as the interactions between let7f-5p and MAPK6, let7f-5p and STAT3 or MAPK6 and STAT3 in pneumonia have not been previously reported. Therefore, to the best of our knowledge, the present study indicated for the first time that the let7f-5p/MAPK6/STAT3 axis may serve an inhibitory role in inflammation in pneumonia.

The present study had two key limitations. The results of the present study demonstrated that let7f-5p expression was significantly decreased in patients with pneumonia and in LPS-induced A549 and WI-38 cells compared with healthy volunteers and control cells, respectively. Therefore, the present study aimed to investigate the effect of let7f-5p overexpression on pneumonia and to assess whether MAPK6 overexpression could rescue the effects of let7f-5p overexpression on pneumonia. The results indicated that let7f-5p inhibited pneumonia-associated inflammation in vitro by targeting MAPK6. However, the effects of MAPK6 knockdown or knockout on inflammation could be similar to the effects mediated by let7f-5p overexpression, but this was not investigated in the present study, thus further investigation is required. Secondly, although the WI-38 cell line is widely used for the study of pneumonia in vitro $(13,38,39)$, a future study using a normal non-cancerous human lung cell line should be performed to verify the results of the present study.

Collectively, the results of the present study suggested that let7f-5p inhibited pneumonia-associated inflammation in vitro by targeting MAPK6 and inactivating the STAT3 signaling pathway. Therefore, let7f-5p may serve as a potential target for anti-inflammatory therapeutic strategies for pneumonia.

\section{Acknowledgements}

Not applicable.

\section{Funding}

The present study was funded by the Respiratory Disease Clinical Research Center of Guizhou Province (grant no. 2016-2907) and the Science and Technology Project of Guizhou Province (grant nos. 2017-1100 and 2019-1195).

\section{Availability of data and materials}

The datasets used and/or analyzed during the present study are available from the corresponding author upon reasonable request.

\section{Authors' contributions}

LX, QS and ZO performed the experiments and analyzed the data. $\mathrm{XZ}$ and $\mathrm{CZ}$ designed the present study and supervised the experiments. $\mathrm{CZ}$ drafted the manuscript. All authors read and approved the final manuscript.

\section{Ethics approval and consent to participate}

The present study was approved by the Ethical Committee of Guizhou Provincial People's Hospital (approval no. 2016011605). The parents or legal guardians of all participants provided written informed consent.

\section{Patient consent for publication}

Not applicable.

\section{Competing interests}

The authors declare that they have no competing interests.

\section{References}

1. Cillóniz C, Torres A, Niederman M, van der Eerden M, Chalmers J, Welte T and Blasi F: Community-acquired pneumonia related to intracellular pathogens. Intensive Care Med 42: 1374-1386, 2016.

2. Korppi M: Diagnosis and treatment of community-acquired pneumonia in children. Acta Paediatr 101: 702-704, 2012.

3. Simonetti AF, Viasus D, Garcia-Vidal C and Carratalà J: Management of community-acquired pneumonia in older adults. Ther Adv Infect Dis 2: 3-16, 2014. 
4. Kim GD: Myricetin inhibits angiogenesis by inducing apoptosis and suppressing PI3K/Akt/mTOR signaling in endothelial cells. J Cancer Prev 22: 219-227, 2017.

5. Yu B, Shen Y, Qiao J and Cui Q: Geniposide attenuates Staphylococcus aureus-induced pneumonia in mice by inhibiting NF- $\kappa$ B activation. Microb Pathog 112: 117-121, 2017.

6. Rojas M, Woods CR, Mora AL, Xu J and Brigham KL: Endotoxin-induced lung injury in mice: Structural, functional, and biochemical responses. Am J Physiol Lung Cell Mol Physiol 288: L333-L341, 2005.

7. Didier D and Jean-Damien R: Acute lung injury and bacterial infection. Clin Chest Med 26: 105-112, 2005.

8. Wong KY, Huang $X$ and Chim CS: DNA methylation of microRNA genes in multiple myeloma. Carcinogenesis 33: $1629-1638,2012$

9. Fei S, Cao L and Pan L: microRNA3941 targets IGF2 to control LPS-induced acute pneumonia in A549 cells. Mol Med Rep 17: 4019-4026, 2018.

10. Abd-El-Fattah AA, Sadik NA, Shaker OG and Aboulftouh ML: Differential microRNAs expression in serum of patients with lung cancer, pulmonary tuberculosis, and pneumonia. Cell Biochem Biophys 67: 875-884, 2013.

11. Huang S, Feng C, Zhai YZ, Zhou X, Li B, Wang LL, Chen W, Lv FQ and Li TS: Identification of miRNA biomarkers of pneumonia using RNA-sequencing and bioinformatics analysis. Exp Ther Med 13: 1235-1244, 2017.

12. Bai D, Han A and Cong S: The effect of down-regulation of CCL5 on lipopolysaccharide-induced WI-38 fibroblast injury: A potential role for infantile pneumonia. Iran J Basic Med Sci 21 : 449-454, 2018.

13. Zhou Z, Zhua Y, Gao G and Zhang Y: Long noncoding RNA SNHG16 targets miR-146a-5p/CCL5 to regulate LPS induced WI-38 cell apoptosis and inflammation in acute pneumonia. Life Sci 228: 189-197, 2019.

14. Wang Q, Li D, Han Y, Ding X, Xu T and Tang B: MicroRNA-146 protects A549 and H1975 cells from LPS-induced apoptosis and inflammation injury. J Biosci 42: 637-645, 2017.

15. Livak KJ and Schmittgen TD: Analysis of relative gene expression data using real-time quantitative PCR and the 2(-Delta Delta C(T)) method. Methods 25: 402-408, 2001

16. Pérez-Herrero E and Fernández-Medarde A: Advanced targeted therapies in cancer: Drug nanocarriers, the future of chemotherapy. Eur J Pharm Biopharm 93: 52-79, 2015.

17. Wang X, Cao L, Wang Y, Wang X, Liu N and You Y: Regulation of let-7 and its target oncogenes (Review). Oncol Lett 3: 955-960, 2012.

18. Tan KS, Choi H, Jiang X, Yin L, Seet JE, Patzel V, Engelward BP and Chow VT: Micro-RNAs in regenerating lungs: An integrative systems biology analysis of murine influenza pneumonia. BMC Genomics 15: 587, 2014.

19. Selvamani A, Sathyan P, Miranda RC and Sohrabji F: An antagomir to microRNA Let7f promotes neuroprotection in an ischemic stroke model. PLoS One 7: e32662, 2012.

20. Wang L, Niu X, Hu J, Xing H, Sun M, Wang J, Jian Q and Yang H: After myocardial ischemia-reperfusion, miR-29a, and Let7 could affect apoptosis through regulating IGF-1. Biomed Res Int 2015: 245412, 2015.

21. Damanakis AI, Eckhardt S, Wunderlich A, Roth S, Wissniowski TT, Bartsch DK and Di Fazio P: MicroRNAs let7 expression in thyroid cancer: Correlation with their deputed targets HMGA2 and SLC5A5. J Cancer Res Clin Oncol 142: $1213-1220,2016$

22. Eguchi A, Franz N, Kobayashi Y, Iwasa M, Wagner N, Hildebrand F, Takei Y, Marzi I and Relja B: Circulating extracellular vesicles and their miR 'Barcode' differentiate alcohol drinkers with liver injury and those without liver injury in severe trauma patients. Front Med (Lausanne) 6: 30, 2019.

23. Lv P, Qiu X, Gu Y, Yang X, Xu X and Yang Y: Long non-coding RNA SNHG6 enhances cell proliferation, migration and invasion by regulating miR-26a-5p/MAPK6 in breast cancer. Biomed Pharmacother 110: 294-301, 2019.
24. Wu J, Zhao Y, Li F and Qiao B: miR-144-3p: A novel tumor suppressor targeting MAPK6 in cervical cancer. J Physiol Biochem 75: 143-152, 2019.

25. Luo M, Sun Q, Zhao H, Tao J and Yan D: Long noncoding RNA NEAT1 sponges miR-495-3p to enhance myocardial ischemia-reperfusion injury via MAPK6 activation. J Cell Physiol 235: 105-113, 2020.

26. Zhang Y, Su Z, Liu HL, Li L, Wei M, Ge DJ and Zhang ZJ: Effects of miR-26a-5p on neuropathic pain development by targeting MAPK6 in in CCI rat models. Biomed Pharmacother 107: 644-649, 2018

27. Liu YC, Ho HC, Lee MR, Lai KC, Yeh CM, Lin YM, Ho TY, Hsiang CY and Chung JG: Early induction of cytokines/cytokine receptors and $\mathrm{Cox} 2$, and activation of $\mathrm{NF}-\mathrm{\kappa B}$ in 4-nitroquinoline 1-oxide-induced murine oral cancer model. Toxicol Appl Pharmacol 262: 107-116, 2012.

28. Khan J, Noboru N, Young A and Thomas D: Pro and antiinflammatory cytokine levels (TNF- $\alpha$, IL-1 $\beta$, IL-6 and IL-10) in rat model of neuroma. Pathophysiology 24: 155-159, 2017.

29. Berg AS, Inchley CS, Fjaerli HO, Leegaard TM, Lindbaek M and Nakstad B: Clinical features and inflammatory markers in pediatric pneumonia: A prospective study. Eur J Pediatr 176: 629-638, 2017.

30. Dai JP, Wang QW, Su Y, Gu LM, Zhao Y, chen XX, Chen C, Li WZ, Wang GF and Li KS: Emodin inhibition of influenza A virus replication and influenza viral pneumonia via the Nrf2, TLR4, p38/JNK and NF-kappaB pathways. Molecules 22: 1754, 2017.

31. Yu Q, Zeng K, Ma X, Song F, Jiang Y, Tu P and Wang X: Resokaempferol-mediated anti-inflammatory effects on activated macrophages via the inhibition of JAK2/STAT3, NF- $\mathrm{BB}$ and JNK/p38 MAPK signaling pathways. Int Immunopharmacol 38: 104-114, 2016

32. Gao H and Ward PA: STAT3 and suppressor of cytokine signaling 3: Potential targets in lung inflammatory responses. Expert Opin Ther Targets 11: 869-880, 2007.

33. Liu G, Wan Q, Li J, Hu X, Gu X and Xu S: Circ_0038467 regulates lipopolysaccharide-induced inflammatory injury in human bronchial epithelial cells through sponging miR-338-3p. Thorac Cancer 11: 1297-1308, 2020.

34. Tan W, Gu Z, Leng J, Zou X, Chen H, Min F, Zhou W, Zhang L and Li G: Let-7f-5p ameliorates inflammation by targeting NLRP3 in bone marrow-derived mesenchymal stem cells in patients with systemic lupus erythematosus. Biomed Pharmacother 118: $109313,2019$.

35. Yao T, Zha D, Hu C and Wu X: Circ_0000285 promotes podocyte injury through sponging miR-654-3p and activating MAPK6 in diabetic nephropathy. Gene 747: 144661, 2020.

36. Li ZH, Wang YF, He DD, Zhang XM, Zhou YL, Yue H, Huang S, Fu Z, Zhang LY, Mao ZQ, et al: Let-7f-5p suppresses Th17 differentiation via targeting STAT3 in multiple sclerosis. Aging (Albany NY) 11: 4463-4477, 2019.

37. Kim SJ, Pham TH, Bak Y, Ryu HW, Oh SR and Yoon DY: Orientin inhibits invasion by suppressing MMP-9 and IL-8 expression via the PKC $\alpha /$ ERK/AP-1/STAT3-mediated signaling pathways in TPA-treated MCF-7 breast cancer cells. Phytomedicine 50: 35-42, 2018.

38. Zhang Y, Zhu Y, Gao G and Zhou Z: Knockdown XIST alleviates LPS-induced WI-38 cell apoptosis and inflammation injury via targeting miR-370-3p/TLR4 in acute pneumonia. Cell Biochem Funct 37: 348-358, 2019.

39. Zhang L, Dong L, Tang Y, Li M and Zhang M: miR-146b protects against the inflammation injury in pediatric pneumonia through MyD88/NF-kB signaling pathway. Infect Dis (Lond) 52: 23-32, 2020 .

This work is licensed under a Creative Commons Attribution-NonCommercial-NoDerivatives 4.0 International (CC BY-NC-ND 4.0) License. 